\title{
Demonstration of Microcantilever Array with Simultaneous Readout Using an In-Plane Photonic Transduction Method
}

\author{
Seunghyun Kim \\ Gregory P. Nordin \\ nordin@byu.edu \\ Ryan Anderson \\ Weisheng $\mathrm{Hu}$ \\ Yusheng Qian
}

See next page for additional authors

Follow this and additional works at: https://scholarsarchive.byu.edu/facpub

Part of the Electrical and Computer Engineering Commons

\section{Original Publication Citation}

W. Hu, R. Anderson, Y. Qian, J. Song, J.W. Noh, S. Kim, G.P. Nordin, "Demonstration of microcantilever array with simultaneous readout using in-plane photonic transduction method," Rev. Sci. Instr. 8, 8511, pp. 1-7 (29). http://link.aip.org/link/?RSINAK/8/8511/1

\section{BYU ScholarsArchive Citation}

Kim, Seunghyun; Nordin, Gregory P.; Anderson, Ryan; Hu, Weisheng; Qian, Yusheng; Song, Jiguo; and Wook Noh, Jong, "Demonstration of Microcantilever Array with Simultaneous Readout Using an In-Plane Photonic Transduction Method" (2009). Faculty Publications. 849.

https://scholarsarchive.byu.edu/facpub/849

This Peer-Reviewed Article is brought to you for free and open access by BYU ScholarsArchive. It has been accepted for inclusion in Faculty Publications by an authorized administrator of BYU ScholarsArchive. For more information, please contact ellen_amatangelo@byu.edu. 


\section{Authors}

Seunghyun Kim, Gregory P. Nordin, Ryan Anderson, Weisheng Hu, Yusheng Qian, Jiguo Song, and Jong Wook Noh 


\title{
Demonstration of microcantilever array with simultaneous readout using an in-plane photonic transduction method
}

\author{
Weisheng Hu, Ryan Anderson, Yusheng Qian, Jigou Song, Jong Wook Noh, \\ Seunghyun Kim, and Gregory P. Nordin \\ Electrical and Computer Engineering, Brigham Young University, Provo, Utah 84602, USA
}

(Received 15 April 2009; accepted 6 July 2009; published online 6 August 2009)

\begin{abstract}
We demonstrate a microcantilever array with an in-plane photonic transduction method for simultaneous readout of each microcantilever. The array is fabricated on a silicon-on-insulator substrate. Rib waveguides in conjunction with a compact waveguide splitter network comprised of trench-based splitters and trench-based bends route light from a single optical input to each microcantilever on the chip. Light propagates down a rib waveguide integrated into the microcantilever and, at the free end of the microcantilever, crosses a small gap. Light is captured in static asymmetric multimode waveguides that terminate in $Y$-branches, the outputs of which are imaged onto an InGaAs line scan camera. A differential signal for each microcantilever is simultaneously formed from the two outputs of the corresponding $Y$-branch. We demonstrate that reasonable signal uniformity is obtained with a scaled differential signal for seven out of nine surviving microcantilevers in an array. (C) 2009 American Institute of Physics.
\end{abstract}

[DOI: $10.1063 / 1.3186735$ ]

\section{INTRODUCTION}

Microcantilevers have been shown to be attractive labelfree nanomechanical sensing devices in which the selectivity is primarily determined by the receptor coating applied to a given microcantilever. ${ }^{1-3}$ Typically either a change in resonance frequency or a change in static deflection is measured to determine adsorption of target molecules by the receptor coating. ${ }^{3}$ Sensing of biological, ${ }^{3-10}$ chemical, ${ }^{1,11-13}$ and environmental contaminant ${ }^{14}$ molecular species has been demonstrated. Two factors that affect the usefulness of microcantilevers in these scenarios are the transduction method for measuring changes in microcantilever properties and the ability to create large compact arrays of microcantilevers. A number of transduction methods have been developed including optical beam deflection, ${ }^{4-6,11}$ piezoresistive, ${ }^{7-10,14,15}$ piezoelectric, ${ }^{12,16}$ capacitive, ${ }^{17,18}$ and optical waveguide techniques. ${ }^{19-21}$ Because of the ready availability of atomic force microscope readout heads, the most widely used of these is the optical beam deflection technique in which a laser beam is reflected off the unclamped end of a microcantilever onto a position-sensitive photodetector. Unfortunately, this technique does not easily scale to simultaneously readout large arrays of microcantilevers, which has led to interest in other transduction methods.

Biological or chemical sensors based on large arrays of microcantilevers can enable simultaneous parallel assays that can increase the efficiency and sensitivity of the sensor and distinguish environmental effects from effects due to the presence of target analytes. ${ }^{4,22-24}$ For example, a number of microcantilevers in an array can be coated with the same receptor to provide detection redundancy for a given target analyte which improves the signal-to-noise ratio of the overall sensor. Moreover, multiple assays for different target ana- lytes increase the overall efficiency of the sensor when dealing with a limited sample volume, and potentially reduce the cost of multiple assays by consolidating them into a single test. Additionally, reference microcantilevers can be included in an array to calibrate out effects due to the ambient sensing environment such as variations in temperature, $p \mathrm{H}$, salt concentration, and fluid flow rate, thereby allowing the sensor to differentiate between changes due to the environment and changes due to adsorption of target analytes. Hence, development of large scale microcantilever arrays is an attractive prospect for sensor applications.

The purpose of this paper is to demonstrate the application of our recently reported ${ }^{25}$ in-plane photonic transduction method to an array of microcantilevers. ${ }^{26,27}$ In our approach, each microcantilever becomes a single-mode rib waveguide that is used in conjunction with a static asymmetric multimode capture waveguide with two outputs to determine the deflection state of the microcantilever through formation of a differential signal. To adapt this technique to an array on a chip, we have developed a compact waveguide splitter network $^{28}$ utilizing trench-based bends ${ }^{29}$ (TBBs) and splitters $^{30}$ (TBSs) to route light from a single optical input to each microcantilever in an array. Such compact splitter networks are crucial to ultimately creating large scale microcantilever arrays because conventional waveguide splitter approaches are prohibitively large given the optical properties of our single-mode rib waveguides. ${ }^{28}$ In this paper we report the first successful integration of a compact splitter network with an array of microcantilevers, and simultaneous readout of the two optical outputs of each microcantilever with an InGaAs line scan camera while externally actuating the microcantilevers. Of the nine surviving released microcantilevers in the array, seven are shown to have comparable differential signal behavior. 
This paper is organized as follows. The design of a microcantilever array with an integrated waveguide splitter network is introduced in Sec. II while array fabrication is discussed in Sec. III. Our experimental approach is presented in Sec. IV and measurement results are discussed in Sec. V.

\section{DESIGN}

Several groups proposed using an embedded waveguide within a microcantilever as an in-plane photonic transduction mechanism. ${ }^{19-21}$ However, in all cases these involve a singlemode capture waveguide to collect light from the end of the microcantilever. As discussed in Ref. 25, this inevitably results in little to no sensitivity near zero deflection where the slope of the captured power as a function of deflection is zero. As demonstrated in Ref. 25, our design eliminates this problem by employing an asymmetric, multimode receiver waveguide with two optical outputs with which a differential signal is formed that is a monotonic function of deflection.

Figure 1(a) shows a schematic of our microcantilever and receiver waveguide geometry, which is fabricated at the top silicon layer of a silicon-on-insulator (SOI) wafer. The microcantilever waveguide consists of a $0.75 \mu \mathrm{m}$ thick, $1.6 \mu \mathrm{m}$ wide ridge surrounded by a slab region that is $0.65 \mu \mathrm{m}$ thick. The result is a rib waveguide that supports a single transverse electric (TE) (electric field in the plane of the silicon layer) mode at a wavelength of $1550 \mathrm{~nm}$. A static receiver waveguide is situated across a small gap $(120 \mathrm{~nm})$ from the end of the microcantilever. It consists of a $3.0 \mu \mathrm{m}$ wide multimode rib waveguide in the silicon layer that has the same ridge height as the multimode waveguide, and a $0.1 \mu \mathrm{m}$ thick amorphous silicon strip loading that is $1.5 \mu \mathrm{m}$ wide and place over half of the rib waveguide. The asymmetric multimode section terminates in a $Y$-branch $1 \times 2$ optical power splitter. Figure 1(b) shows the simulated normalized optical power in each output, $P_{1}$ and $P_{2}$, as a function of microcantilever deflection. The slight offset, $\Delta$, between the peaks of the output power profiles due to the receiver waveguide asymmetry makes it possible to use these signals to form a differential signal, $\eta$, defined as

$$
\eta=\frac{P_{2}-P_{1}}{P_{2}+P_{1}},
$$

that is monotonic and nearly linear across the measurement range of interest, as shown in Fig. 1(c). This avoids the low sensitivity region that exists in the measurement range when a simple single-mode capture waveguide is used. Note also that unlike the readout with optical beam deflection, our transduction method is suitable even for opaque solutions since the optical beam is in solution only as it traverses the gap between the end of the microcantilever and the static receiver waveguide, and this path length is extremely small resulting in minimal attenuation.

Using our in-plane transduction method to create a large array of microcantilevers requires sourcing light into each microcantilever waveguide. This is accomplished with a splitter network using TBBs and TBSs similar to those reported in Refs. 28-30. As discussed in Ref. 28, we use TBSs and TBBs with a bend angle of $105^{\circ}$ to achieve a 50/50

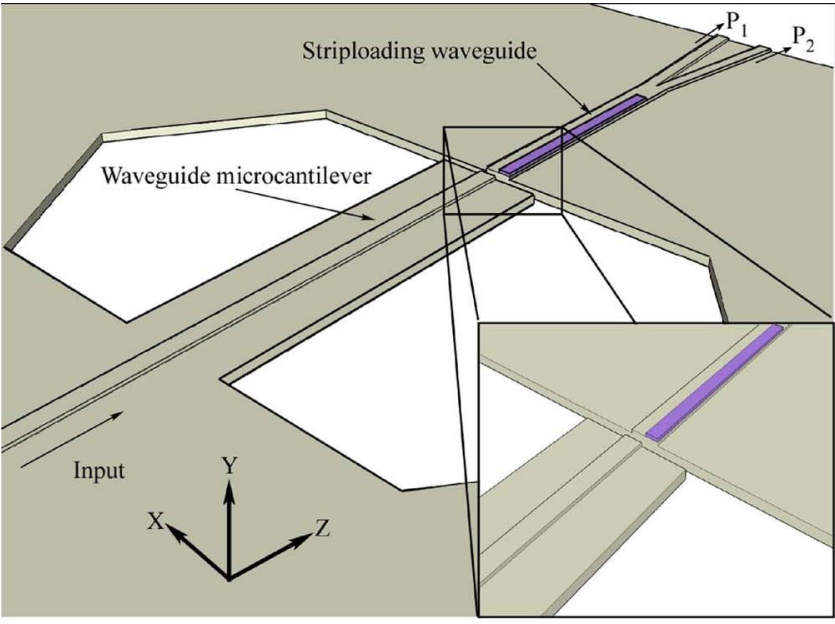

(a)

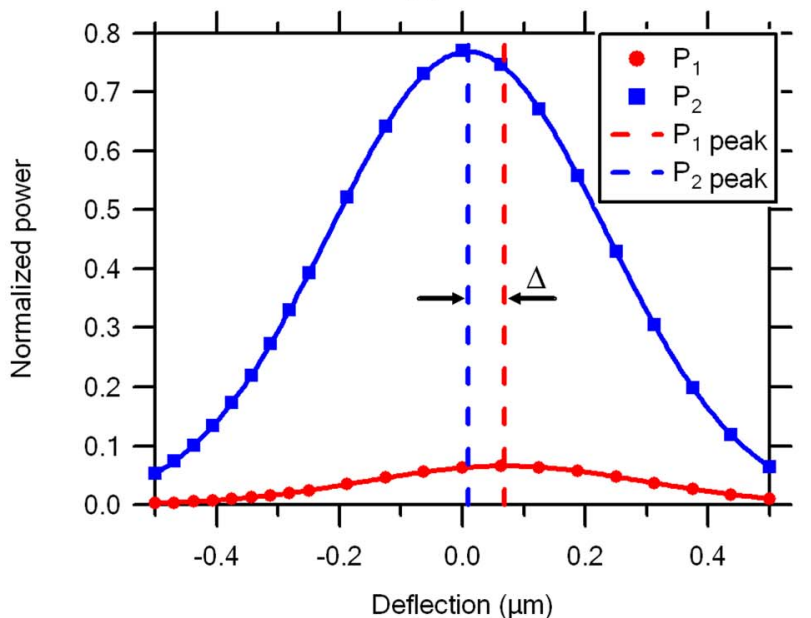

(b)

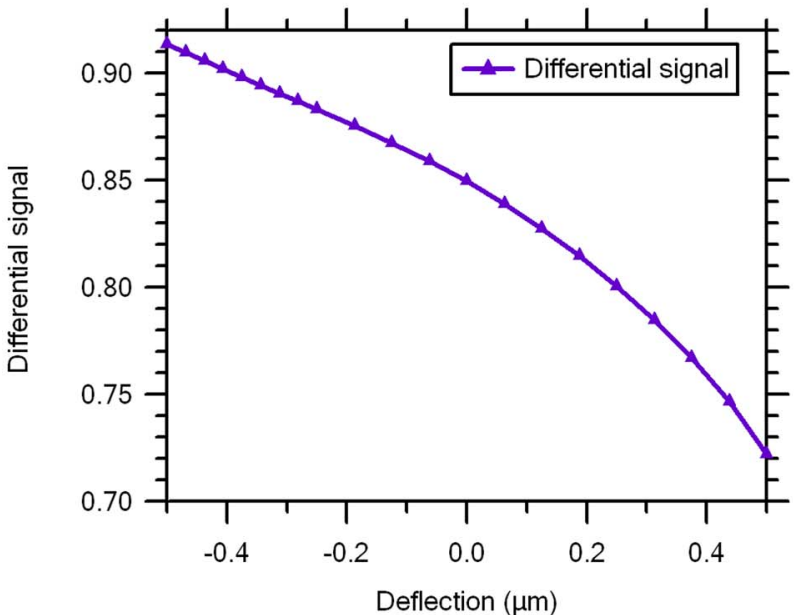

(c)

FIG. 1. (Color online) (a) Schematic layout of photonic microcantilever with asymmetric multimode receiver waveguide (after Ref. 25). Simulation results for (b) output power and (c) differential signal as a function of microcantilever deflection. Simulations are performed with FIMMWAVE/PROP by Photon Design.

splitting ratio for the splitters. The waveguide layout on the silicon die was originally designed to accommodate a $1 \times 32$ network with a $100 \mu \mathrm{m}$ cantilever spacing and a $50 \mu \mathrm{m}$ output waveguide spacing (two outputs per cantilever). However, to simplify the readout optics, we choose to implement a $1 \times 16$ network, as shown in Fig. 2, sourcing 


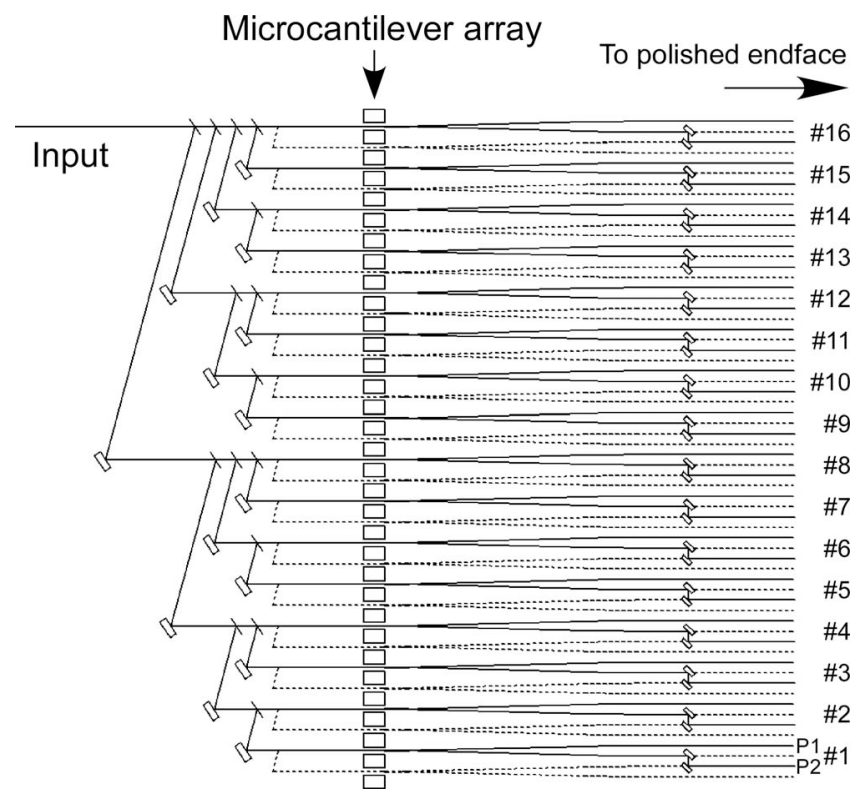

FIG. 2. Schematic layout of waveguide splitter network and microcantilever array. The solid lines represent waveguides that are optically sourced and correspond to active microcantilevers in the array; the dashed lines represent waveguides that are not used and correspond to inactive microcantilevers in the array. The numbers on the far right indicate the numbering convention for the active microcantilevers.

light into every other microcantilever, which produces a $100 \mu \mathrm{m}$ output spacing of the illuminated output waveguides. This also requires placing two TBBs on one of the output waveguides of each microcantilever to reroute the $P_{2}$ signal.

\section{FABRICATION}

The waveguide splitter network, microcantilevers, and asymmetric receiver waveguides are fabricated using processes similar to those reported in Refs. 25 and 28-30. Fabrication begins with a $100 \mathrm{~mm}$ SOI wafer that has a $0.75 \mu \mathrm{m}$ single crystal silicon layer and a $3 \mu \mathrm{m}$ buried oxide layer. Waveguides and cantilevers are defined in separate photolithography steps in a contact mask aligner, each of which is followed by a silicon etch in an inductively coupled plasma reactive ion etcher (ICP RIE) (Surface Technology Systems). During waveguide patterning, alignment marks are also patterned for use in subsequent lithography steps. Next, the wafer is diced into discrete die. An individual die is further processed by patterning the trenches for the TBBs and TBSs in the waveguide splitter network and $P_{2}$ output waveguides, as shown in Figs. 3(a)-3(c), and $120 \mathrm{~nm}$ gaps for the free ends of the microcantilevers, as shown in Fig. $3(\mathrm{~d})$. This is done by electron beam lithography (EBL) with a Nanometer Pattern Generation System (JC Nabity NPGS) and field emission environmental scanning electron microscope (SEM) (FEI/Philips XL30 ESEM-FEG). A water soluble conductive polymer (aquaSAVE53za) is spin coated on top of the electron-beam resist (ZEP 520A) to prevent charging during EBL. The trenches and gaps are etched in an ICP RIE with a fluorine-based etch chemistry. After stripping of the e-beam resist, an additional EBL step is done to define the strip loading on the multimode waveguides, followed by sputtering of amorphous silicon and lift-off. Figure 3(e) shows a close-up image of the strip loading on the multimode receiver waveguide. SU-8 is spin coated to fill the bend and splitter trenches and also to act as the waveguide upper cladding over most of the chip (except the microcantilever region). The input and output faces of the die are polished to facilitate optical coupling through the waveguide end faces. Etching in hydrofluoric (HF) acid followed by critical point drying (Tousimis Autosamdri 815B) removes the buried oxide and releases the microcantilevers.

\section{EXPERIMENT}

To characterize the differential signals from the array of microcantilevers, we have developed an experimental setup that is capable of actuating all microcantilevers simultaneously while also collecting the $P_{1}$ and $P_{2}$ optical power levels. The critical aspect of this experimental approach is patterning a SU-8 polymer layer on the top of each microcantilever and heat treating the sample to deliberately cause the microcantilevers to deflect due to compressive stress induced by thermally driven epoxy cross-linking.

Figure 4 shows the general scheme for experimentally measuring the differential signal for each microcantilever as a function of deflection. Light from a fiber-coupled superluminescent light emitting diode with a center wavelength of $1550 \mathrm{~nm}$ is propagated through a polarization-maintaining fiber oriented so that the output polarization is matched to the TE mode of the optical waveguide. Light from the fiber is butt coupled to the chip's input waveguide, from which it is directed through each microcantilever by the waveguide splitter network. Actuation of the microcantilevers is accomplished with a glass piece attached to a piezotranslator (Physik Instrumente, P-841.30). The glass is $500 \mu \mathrm{m}$ thick and cut in a tapered shape $\sim 4 \mathrm{~cm}$ in length and end widths of 10 and $2 \mathrm{~mm}$. The $2 \mathrm{~mm}$ wide end physically contacts the microcantilever array and spans a maximum of ten of the optically sourced cantilevers. We polished this end to remove any defects so that the deflection from cantilever to cantilever is as uniform as possible. Two goniometer stages allow us to adjust the angle and position of the glass piece so that its edge is parallel to the row of microcantilevers. The glass piece is positioned to actuate microcantilevers 5-14. Microcantilevers 1, 2, 15, and 16 do not have gaps patterned such that they are not released (i.e., doubly clamped beams). Instead, their purpose is to provide static output sources for aligning the readout optics such that light from a single output waveguide is imaged to a single pixel on the InGaAs linear array. Unfortunately, microcantilevers 3, 4, and 12 were broken during an early test scan and so do not provide any data. Hence 9 of the 12 released microcantilevers contributed to our measurements.

The InGaAs digital line scan camera (Goodrich SU512LSE) is set to sample at a line scan rate of $1440 \mathrm{~Hz}$ with an exposure time of $0.48 \mathrm{~ms}$. Readout optics image the waveguide outputs to every fourth pixel of the InGaAs linear array. The total piezoactuator scan range for the experiment is set to $3 \mu \mathrm{m}$, taken in $0.05 \mu \mathrm{m}$ steps. At each step 800 line scans are averaged to obtain the average power for each 


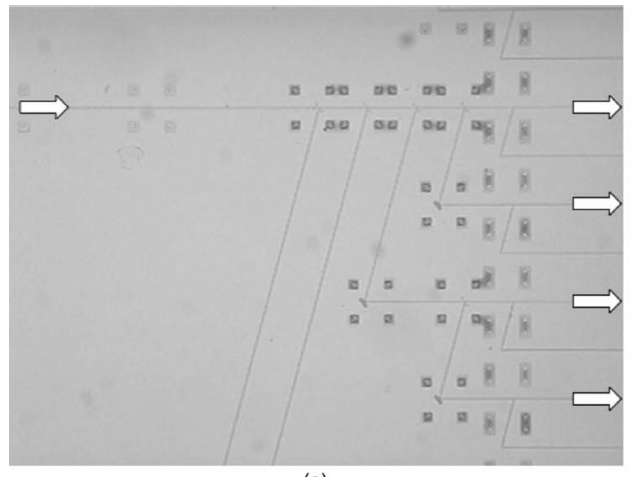

(a)

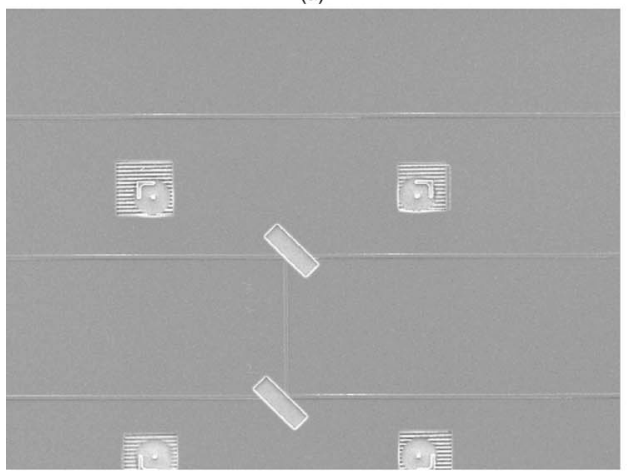

(c)

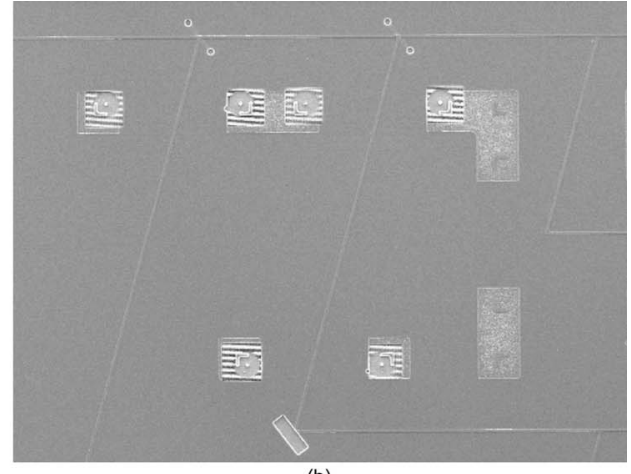

(b)

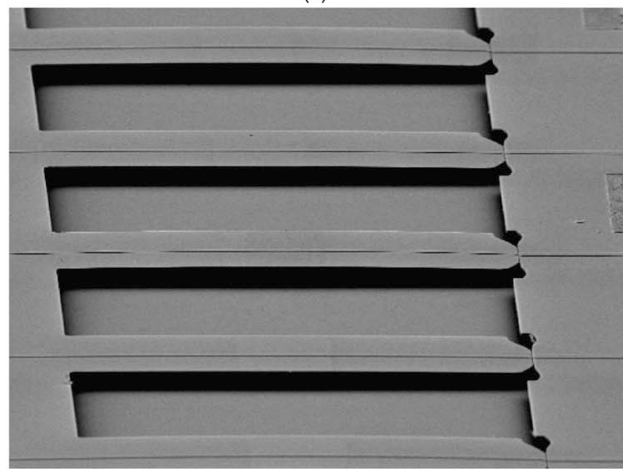

(d)

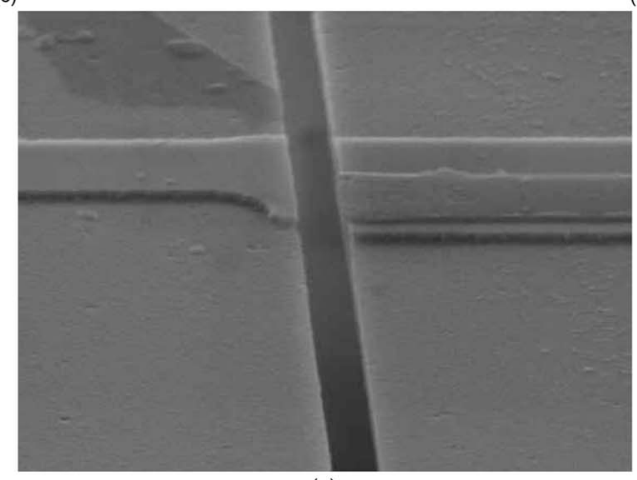

(e)

output waveguide at that piezoactuator position. The piezoactuator position is converted into actual microcantilever deflection based on the ratio of the total microcantilever length to the position along the microcantilever at which the glass piece makes contact.

\section{RESULTS}

Measured $P_{1}$ and $P_{2}$ data for each of the nine unbroken released microcantilevers are shown in Figs. 5(a) and 5(b), respectively. Gaussian fits to the measured data are also pro-

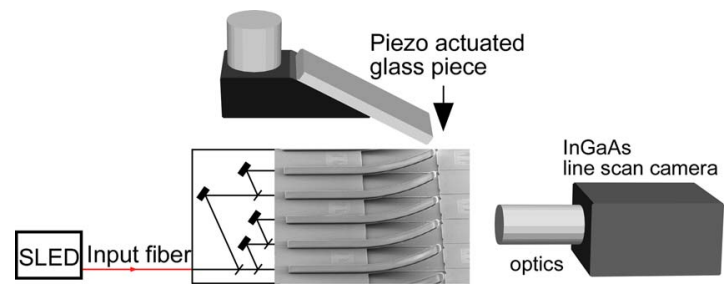

FIG. 4. (Color online) Schematic of the experimental setup showing network array, the bent up cantilevers, the glass piece for pushing down on the cantilevers, and the imaging camera for simultaneous readout. vided. Note that the peak positions of the $P_{1}$ data do not occur at the same piezo position, and similarly for the $P_{2}$ data. Figure 5(c) shows the relative $P_{1}$ and $P_{2}$ peak positions for each microcantilever. Systematic variation of the peak positions is clearly present, indicating that the edge of the glass piece used to push down the microcantilevers is slightly bowed, probably due to the polishing process used in its fabrication. Note also in Fig. 5(c) that the difference in $P_{1}$ and $P_{2}$ peak positions is much smaller for microcantilevers 8 and 9 than for the other microcantilevers. Quantitative data are given in the second column of Table I. As noted in Ref. 25 , the larger $\Delta$ is, the greater the range one expects to see for the differential signal. Consequently, we expect microcantilevers 8 and 9 to show poor differential signal characteristics.

Correcting for the systematic variation in the measured $P_{1}$ and $P_{2}$ peak positions and converting piezo position to deflection, the differential signal for each microcantilever is shown in Fig. 5(d). As expected, microcantilever 8 shows almost no variation in the differential signal since its $\Delta$ is so small. Also, the differential signal for microcantilever 9 is anomalous in that it is not a monotonic function of deflec- 

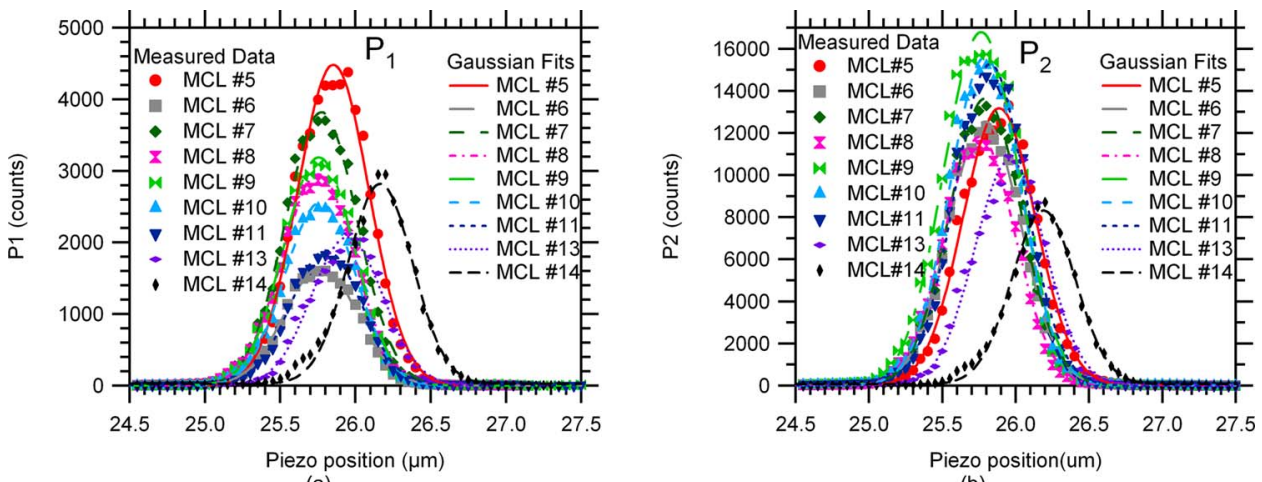

(a)

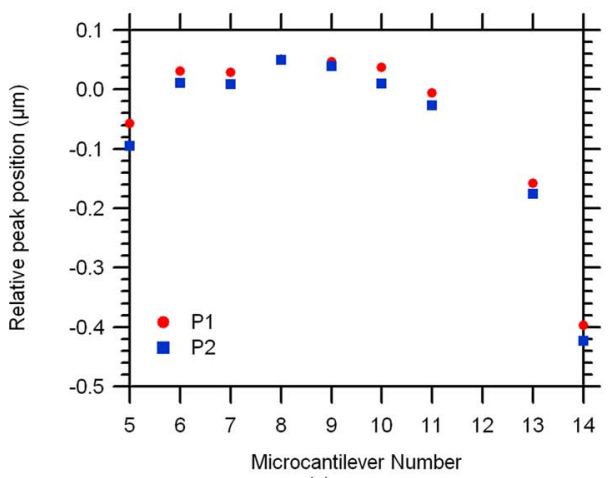

(c)

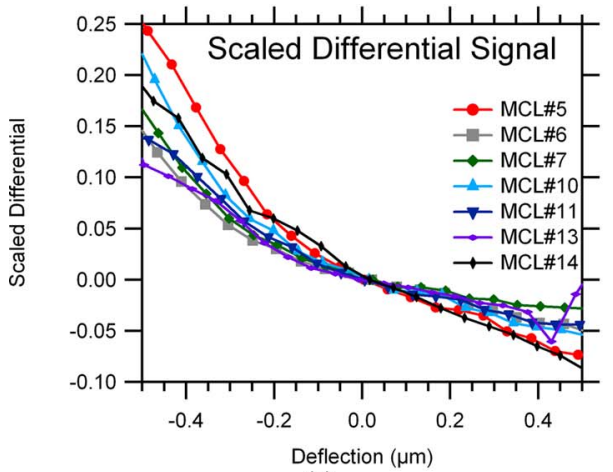

(e)

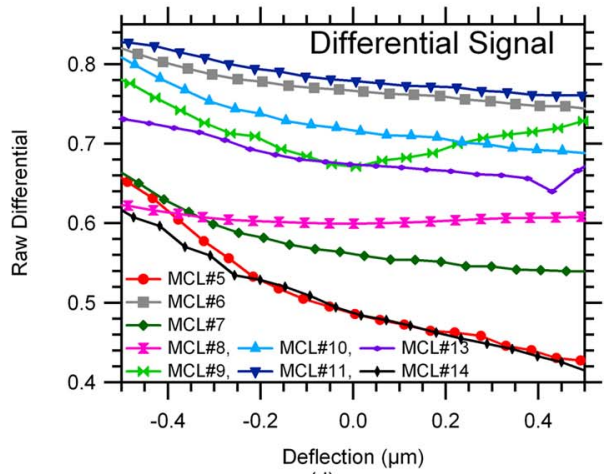

(d)

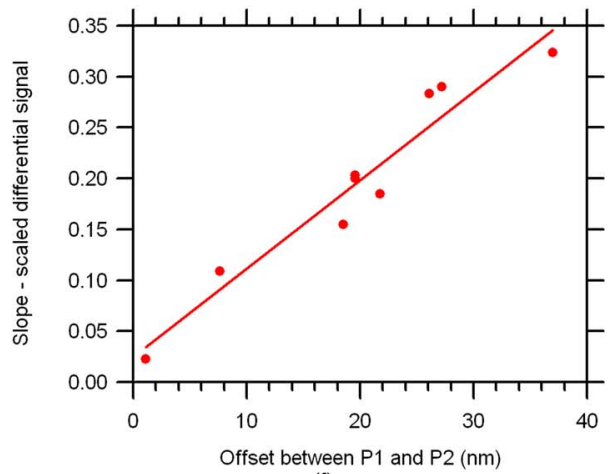

FIG. 5. (Color online) Measured (a) $P_{1}$ and (b) $P_{2}$ signals in units of electron counts (proportional to the total flux incident on the camera pixels) of each microcantilever as a function of piezoactuator position. (c) Relative position of $P_{1}$ and $P_{2}$ Gaussian fit peaks for each microcantilever. (d) Differential and (e) scaled differential signals from the $P_{1}$ and $P_{2}$ data. (f) Slope of each scaled differential signal plotted against the difference in the corresponding $P_{1}$ and $P_{2}$ peak positions. tion. So far our investigations have not revealed why this is the case. Nonetheless, the other microcantilevers show a monotonic dependence on deflection with reasonable slope. Note, however, that most of the curves are separated vertically from each other. As discussed in Ref. 25, this occurs when the ratio of the measured $P_{1}$ and $P_{2}$ peak values is different, which can have a variety of causes such as wave-

TABLE I. Difference in $P_{1}$ and $P_{2}$ peak positions $(\Delta), P_{1} / P_{2}$ peak ratio, and average slope of scaled differential signal for each microcantilever.

\begin{tabular}{cccc}
\hline \hline MCL & $\begin{array}{c}\text { Peak difference } \\
(\mathrm{nm})\end{array}$ & $P_{1} / P_{2}$ peak ratio & $\begin{array}{c}\text { Slope } \\
\left(\mu \mathrm{m}^{-1}\right)\end{array}$ \\
\hline 5 & 37.0 & 0.34 & 0.324 \\
6 & 19.6 & 0.13 & 0.203 \\
7 & 19.6 & 0.28 & 0.200 \\
8 & 1.1 & 0.25 & 0.023 \\
9 & 7.6 & 0.19 & 0.109 \\
10 & 27.2 & 0.16 & 0.290 \\
11 & 21.8 & 0.12 & 0.185 \\
13 & 18.5 & 0.19 & 0.155 \\
14 & 26.1 & 0.34 & 0.283 \\
\hline \hline
\end{tabular}

guide defects and flaws in the polished waveguide end faces that induce additional loss. As seen in the third column of Table I, there is a wide variation in these ratios in the measured data. Nonetheless, several pairs of microcantilevers have similar peak ratio values (for example, microcantilevers 5 and 14, and 6 and 11) and their differential signal curves are correspondingly close to each other.

The vertical offset in differential signals makes direct comparison of differential signals between microcantilevers in an array problematic for sensing scenarios. We therefore formulate an alternate differential signal that is more useful for microcantilever arrays in that it uses a simple scaling parameter to largely remove the variation caused by different $P_{1} / P_{2}$ ratios. We define this scaled differential signal, $\eta_{\text {scaled }}$, as

$$
\eta_{\text {scaled }}=\frac{P_{2}-\alpha P_{1}}{P_{2}+\alpha P_{1}},
$$

in which the scaling factor, $\alpha$, is defined as 


$$
\alpha=\frac{P_{20}}{P_{10}},
$$

where $P_{20}$ and $P_{10}$ are the measured output powers at an arbitrary reference deflection for any given microcantilever. The reference deflection should ideally be chosen where both $P_{1}$ and $P_{2}$ have strong signals, preferably at or near zero deflection so that the scaling factor is not strongly affected by noise. In practice, for sensor applications $P_{10}$ and $P_{20}$ can be the measured values of $P_{1}$ and $P_{2}$ prior to introduction of the sample to be analyzed. Extensive analysis shows that this has the effect of rescaling $P_{1}$ so that all cantilevers have roughly the same the $P_{2}$ to $P_{1}$ peak ratio, and ensures that the scaled differential signals of each cantilever have the same value at the reference deflection. The scaled differential signal can therefore compensate for any additional optical loss in the output waveguides after the $Y$-branch splitter that affects one output more than the other.

The effectiveness of using the scaled differential signal is noticeable when comparing the scaled differential signals in Fig. 5(e) to the differential signals in Fig. 5(d). [Note that we have chosen not to show results for microcantilevers 8 and 9 in Fig. 5(e) because $\Delta$ is so small for each of them.] The scaled differential signals have no significant vertical separation. Instead, the remaining variation is primarily a difference in the signal range of each scaled differential signal as evidenced by the different end points of each curve over the $\pm 0.5 \mu \mathrm{m}$ deflection range shown in the graph. This variation can be captured as an average slope for each curve, which is tabulated in the fourth column of Table I. Note that the slope is directly correlated with $\Delta$, as shown in Fig. 5(f), where a linear relationship is evident. Hence, with the use of a scaled differential signal, improving the uniformity of the optical responses in a microcantilever array that uses our in-plane photonic readout method is primarily a matter of improving the uniformity of $\Delta$ for the microcantilevers. We are currently investigating how to make such improvements. Nonetheless, the scaled differential signal results shown in Fig. 5(e) demonstrate that a microcantilever array can be readout simultaneously with reasonable uniformity using our in-plane photonic transduction method.

A further consideration is that the maximum line scan frequency of our InGaAs line scan camera is $4266 \mathrm{~Hz}$. It is therefore suited to measurement of microcantilever deflection rather than shift in resonance frequency. Measurement of deflection (commonly referred to as static mode operation) is particularly appropriate for microcantilever sensors operating in liquid, where viscous damping dramatically broadens the resonance frequency response thereby limiting the minimum resonance frequency change that can be measured. Noise in a static mode microcantilever readout system can be characterized by the minimum detectable deflection (MDD), which is defined as the amount of deflection corresponding to a signal-to-noise ratio of one. The MDD for the measurements reported in this paper is $183 \mathrm{pm}$ for a $1 \mathrm{~Hz}$ time-averaged bandwidth.

Sensitivity (i.e., signal change per unit deflection, typically measured in inverse nanometers) is an effective means of comparing microcantilever readout methods. Note that this is just the slope of the measurement response. In our case, the average sensitivity is $2.3 \times 10^{-4} \mathrm{~nm}^{-1}$. This is comparable to the sensitivity obtained with optical beam deflection $^{31,32}$ and two orders of magnitude larger than for piezoresistive readout, ${ }^{14,33-35}$ which are the two most widely used microcantilever readout techniques. Hence, in-plane photonic transduction is attractive in that it maintains the sensitivity of optical beam deflection while being scalable to readout arrays of microcantilevers.

\section{CONCLUSION}

In summary, we have demonstrated the integration of a compact waveguide splitter network with a waveguide microcantilever array, and simultaneous microcantilever readout with an InGaAs line scan camera. The fabricated waveguide splitter network sources light into 16 microcantilevers. Asymmetric, multimode receiver waveguides feed into $Y$-branch splitters that give two outputs for each microcantilever. The output light is imaged onto a line scan camera to enable simultaneous readout of all microcantilevers in the array. Use of a scaled differential signal yields reasonable correspondence of the signals from the seven of the nine surviving released microcantilevers in the array. We are now seeking to further improve the response uniformity and to characterize much larger microcantilever arrays (32 and 64 microcantilevers).

\section{ACKNOWLEDGMENTS}

This work was supported in part by the NSF under Grant Nos. ECS-0602261 and IIS-0641973, and the DARPA under Grant No. N66001-0408933.

${ }^{1}$ T. Thundat, E. A. Wachter, S. L. Sharp, and R. J. Warmack, Appl. Phys. Lett. 66, 1695 (1995).

${ }^{2}$ R. Raiteri, M. Grattarola, and R. Berger, Mater. Today 5, 22 (2002).

${ }^{3}$ P. S. Waggoner and H. G. Craighead, Lab Chip 7, 1238 (2007).

${ }^{4}$ J. Fritz, M. K. Baller, H. P. Lang, H. Rothuizen, P. Vettiger, E. Meyer, H.-J. Güntherodt, Ch. Gerber, and J. K. Gimzewski, Science 288, 316 (2000).

${ }^{5}$ G. Wu, R. H. Datar, K. M. Hansen, T. Thundat, R. J. Cote, and A. Majumdar, Nat. Biotechnol. 19, 856 (2001).

${ }^{6}$ R. Bashir, J. Z. Hilt, O. Elibol, A. Gupta, and N. A. Peppas, Appl. Phys. Lett. 81, 3091 (2002).

${ }^{7}$ A. M. Moulin, S. J. O'Shea, and M. E. Welland, Ultramicroscopy 82, 23 (2000)

${ }^{8}$ K. M. Hansen and T. Thundat, Methods 37, 57 (2005).

${ }^{9}$ L. G. Carrascosa, M. Moreno, M. Álvarez, and L. M. Lechuga, TrAC, Trends Anal. Chem. 25, 196 (2006).

${ }^{10}$ J. Zhang, H. P. Lang, F. Huber, A. Bietsch, W. Grange, U. Certa, R. McKendry, H.-J. Güntherodt, M. Hegner, and Ch. Gerber, Nat. Nanotechnol. 1, 214 (2006)

${ }^{11}$ H. P. Lang, R. Berger, F. Battiston, J.-P. Ramseyer, E. Meyer, C. Andreoli, J. Brugger, P. Vettiger, M. Despont, T. Mezzacasa, L. Scandella, H.-J. Güntherodt, Ch. Gerber, and J. K. Gimzewski, Appl. Phys. A: Mater. Sci. Process. 66, S61 (1998)

${ }^{12}$ J. D. Adams, G. Parrott, C. Bauer, T. Sant, L. Manning, M. Jones, B. Rogers, D. McCorkle, and T. L. Ferrell, Appl. Phys. Lett. 83, 3428 (2003).

${ }^{13}$ L. Fadel, F. Lochon, I. Dufour, and O. Français, J. Micromech. Microeng. 14, S23 (2004).

${ }^{14}$ A. Boisen, J. Thaysen, H. Jensenius, and O. Hansen, Ultramicroscopy 82, $11(2000)$

${ }^{15}$ P. A. Rasmussen, J. Thaysen, O. Hansen, S. C. Eriksen, and A. Boisen, Ultramicroscopy 97, 371 (2003).

${ }^{16}$ J. D. Adams, B. Rogers, L. Manning, Z. Hu, T. Thundat, H. Cavazos, and S. C. Minne, Sens. Actuators, A 121, 457 (2005). 
${ }^{17}$ C. L. Britton, R. L. Jones, P. I. Oden, Z. Hu, R. J. Warmack, S. F. Smith, W. L. Bryan, and J. M. Rochelle, Ultramicroscopy 82, 17 (2000).

${ }^{18}$ J. Amírola, A. Rodríguez, L. Castañer, J. P. Santos, J. Gutiérrez, and M. C. Horrillo, Sens. Actuators B 111, 247 (2005).

${ }^{19}$ K. E. Burcham, G. N. D. Brabander, and J. T. Boyd, Proc. SPIE 1793, 12 (1993).

${ }^{20}$ S. Wu and H. J. Frankena, Proc. SPIE 1793, 83 (1993).

${ }^{21}$ M. Nordström, D. A. Zauner, M. Calleja, J. Hubner, and A. Boisen, Appl. Phys. Lett. 91, 103512 (2007)

${ }^{22}$ R. Zhang, A. Best, R. Berger, S. Cherian, S. Lorenzoni, E. Macis, R. Raiteri, and R. Cain, Rev. Sci. Instrum. 78, 084103 (2007).

${ }^{23}$ R. McKendry, J. Y. Zhang, Y. Arntz, T. Strunz, M. Hegner, H. P. Lang, M. K. Baller, U. Certa, E. Meyer, H.-J. Güntherodt, and Ch. Gerber, Proc. Natl. Acad. Sci. U.S.A. 99, 9783 (2002).

${ }^{24}$ M. Yue, H. Lin, D. E. Dedrick, S. Satyanarayana, A. Majumdar, A. A. Bedekar, J. W. Jenkins, and S. Sundaram, J. Microelectromech. Syst. 13, 290 (2004).

${ }^{25}$ J. W. Noh, R. Anderson, S. Kim, J. Cardenas, and G. P. Nordin, Opt. Express 16, 12114 (2008).
${ }^{26}$ G. P. Nordin, J. W. Noh, Y. Qian, J. Song, R. Anderson, and S. Kim, International Workshop on Nanomechanical Sensors, Montreal, Canada, 28-30 May 2007 (unpublished).

${ }^{27}$ G. P. Nordin, J. W. Noh, and S. Kim, Proc. SPIE 6447, 64470J (2007).

${ }^{28}$ Y. Qian, J. Song, S. Kim, W. Hu, and G. P. Nordin, Opt. Express 16, 4981 (2008).

${ }^{29}$ Y. Qian, S. Kim, J. Song, and G. P. Nordin, Opt. Express 14, 6020 (2006).

${ }^{30}$ Y. Qian, J. Song, S. Kim, and G. P. Nordin, Opt. Express 15, 16712 (2007).

${ }^{31}$ C. Kocabas and A. Aydinli, IEEE Sens. J. 5, 411 (2005).

${ }^{32}$ K. Zinoviev, C. Dominguez, J. A. Plaza, V. J. C. Busto, and L. M. Lechuga, J. Lightwave Technol. 24, 2132 (2006).

${ }^{33}$ R. L. Gunter, R. Zhine, W. G. Delinger, K. Manygoats, A. Kooser, and T. L. Porter, IEEE Sens. J. 4, 430 (2004).

${ }^{34}$ J. Thaysen, A. D. Yalcinkaya, P. Vettiger, and A. Menon, J. Phys. D: Appl. Phys. 35, 2698 (2002).

${ }^{35}$ X. Yu, Y. Tang, H. Zhang, T. Li, and W. Wang, IEEE Sens. J. 7, 489 (2007). 